\title{
Computer approximation of air gauge dynamic characteristics using the sine input test rig
}

\author{
Michał Jakubowicz' ${ }^{1}$, Mirosław Rucki ${ }^{2}$, and Zbigniew Siemiątkowski,** \\ ${ }^{1}$ Poznan University of Technology, Faculty of Mechanical Engineering and Management, Institute of Mechanical Technology, \\ Pl. Marii Skłodowskiej-Curie 5, 60-965 Poznań, Poland \\ ${ }^{2}$ Kazimierz Pulaski University of Technology and Humanities, Faculty of Mechanical Engineering, ul. Stasieckiego 54, 26-600 \\ Radom, Poland
}

\begin{abstract}
The paper describes the test rig dedicated for air gauge dynamical characteristics assessment. The computerised system enables measurement of the amplitudes of back-pressure $p_{k}$ dependent on the input signal circular frequency $\omega$. Dedicated software performs full control on the calibration procedure, which consists of setting a rotational speed and registration of measuring signal, and further data processing. Circular frequency $\omega$ was gradually changed with the appropriate step, in order to obtain a series of frequencies in the range from 0.1 to $20 \mathrm{~Hz}$. The response of a measurement system was registered as a sinusoidal curve which after smoothening and interpolation procedures provided an amplitude-frequency graph with its main characteristics, such as time constant and the frequency $f_{0.95}$ that generated dynamic error 5\%. It was demonstrated that sine input dynamic calibration corresponds with real conditions of the inprocess measurement with air gauges.
\end{abstract}

\section{Introduction}

The air gauges are known for almost a century as cheap and precise measurement devices [1] with the resolution of $0.1 \mu \mathrm{m}[2]$. Their work principle is to convert the dimension represented by a slot width to the airflow characteristics. Some new propositions apply time conversion of the linear dimension with the air gauges [3]. Nowadays, the most commonly used type is the back-pressure air gauge with electronic conversion of the pressure signal, so it may be incorporated into an extended computerised quality control system [4].

Non-contact measurement and calibration methods are highly appreciated in the laboratory and industrial practice $[5,6]$. In some cases, air gauges enable to perform a measurement in few seconds, while a similar measurement e.g. with a vernier calliper may take up to 1 min [7]. Even though flow-through phenomena may reduce the dynamics of the air gauges [8], recent researches proved that it is possible to obtain first-order dynamical characteristics [9].

Test rigs are equipped with computers performing detailed analyses of the air gauges $[10,11]$. Improving computational capacities stimulates continuous investigations on air gauges dynamic characteristics $[12$, 13].

Dynamic calibration determines the relationship between an input of known dynamic behaviour and the measurement system output. Usually, such calibrations involve applying either a sinusoidal signal or a step change as the known input signal [14]. The present paper describes the computerised test rig dedicated for air gauge dynamical characteristics assessment. It is very important that the dynamic calibration conditions are close to the real conditions of measurement with air gauges, such as roundness assessment [15] or in-situ measurement [16]. The presented test rig applies a sine input and performs the necessary calculations to assess the dynamic characteristics of the examined air gauge.

\section{Experimental rig}

The amplitude-frequency graph provides information regarding how the input signal frequency affects the output signal amplitude. In the case of the air gauges, where the output signal is the back-pressure $p_{k}$, the function of its amplitude is $A_{p k}\left(f_{i}\right)$. Thus, the experimental rig had to be equipped with the devices that generate cyclical sinusoidal input signal (slot width $s$ ), measure the back-pressure $p_{k}$, and perform calculations of the response signal. Assuming that the $5 \%$ reduction of amplitude is usually acceptable, as a critical value $f_{0.5}$ was calculated to represent the maximal input frequency that causes the reduction on this level.

Figure 1 presents the overall view of the test rig, and Figure 2 explains its structure [17]. Main elements of the system listed in the Figure are as follows:

1 - piezoresistive pressure transducer Kistler 4043A2;

2 - examined physical model of the air gauge;

3 - reducer with the filter SMC AW20-A;

4 - pressure calibration unit Fluke 717 30G;

5 - power supply PS3003;

6 - pressure stabiliser SMC IR2000-F02;

7 - computer (software LabVIEW Signal Express);

* Corresponding author: z.siemiatkowski@uthrad.pl 
8 - eccentric shaft of diameter $\varnothing 50 \mathrm{~mm}$ and known eccentricity $e$;

9 - amplifier Kistler 4618 A2;

10 - air gauge positioning screw;

11 - position indicator;

12 - motor for eccentric rotation Maxon DC;

13 - microprocessor system NI-DAQ-9172 with the measurement module NI 9234.

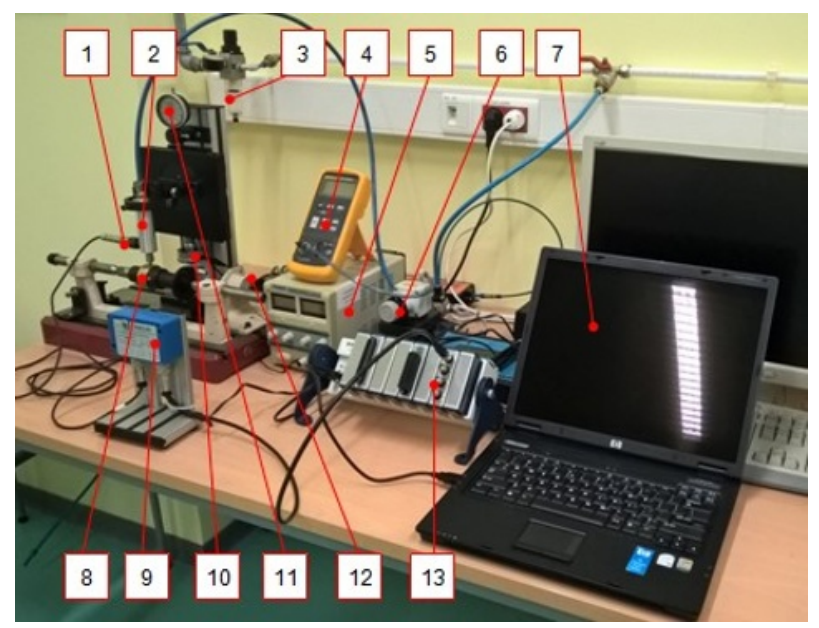

Fig. 1. Test rig for the dynamic sine input calibration of the air gauges (explained in the text above).

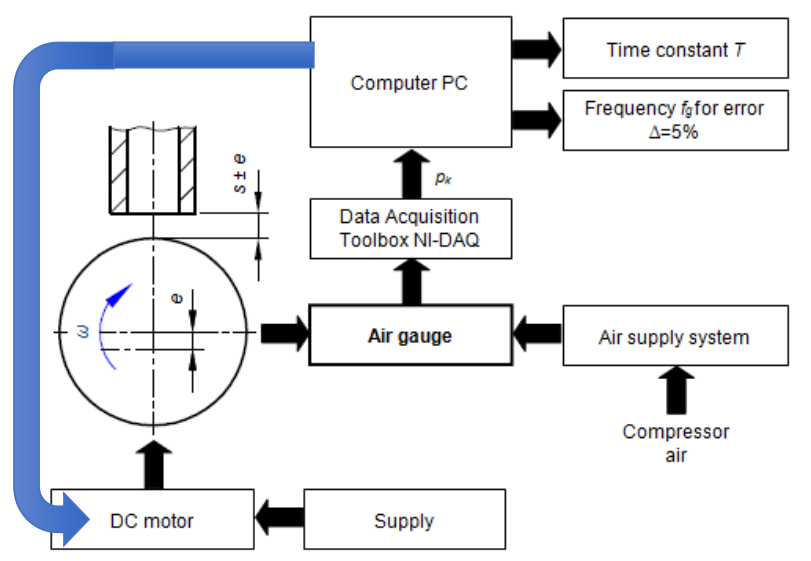

Fig. 2. Structure of the test rig.

The test rig was equipped with a set of elements that enabled adjustment of the position of the air gauge measuring nozzle related to the cylindrical surface of the rotating eccentric shaft. The latter was serving as a flapper, and its eccentricity generated cyclic change of the measurement slot $s(t)$ in the range from $s-e$ to $s+e$. The rotational speed of the shaft was controlled by the programme through the drive system with the DC supplied motor. A gradual increase in the motor voltage supply provided stable rotation of the eccentric shaft, which generated cyclic slot width changes with the preset frequency. Circular input frequency $\omega$ was set with the appropriate step, in order to gain a series of input frequencies $f_{i}$ in the range from 0.1 to $10 \mathrm{~Hz}$, with the step of $0.2 \mathrm{~Hz}$.

The measurement data were registered with NI 9234 measurement module delivered by National Instruments using LabVIEW software.

\section{Theoretical background}

In the unsteady states of air gauge work, the following processes may be assumed:

- air flow through the nozzles may be viewed as independent of the inertia forces,

- gas flow in the air gauge measuring chamber can be treated as isothermal,

- all characteristics that define the air flow in the measuring chamber may be changed, in particular, feeding pressure $p_{z}$, pressure of the air flow at the outer edge of the nozzle $p_{b}$, effective intersections of the nozzles that determine the difference between theoretical and actual flows, etc.

Let us analyse the changes of the air mass in the measuring chamber between the inlet and outlet nozzles. The dynamics of the mass flow $q$ of the air contained in the volume $V_{k}$ of cascade depends on the difference between the mass flow in the inlet nozzle $q_{w}$ and the one leaving the chamber through the flapper-nozzle area $q_{p s}$ :

$$
\frac{d q(t)}{d t}=q_{w}(t)-q_{\mathrm{ps}}(t)
$$

Airflow values $q_{w}$ and $q_{\mathrm{ps}}$ can be defined by the Saint Venant - Wenzel equations. The mass flow $q$ is dependent on measuring pressure $p_{k}$ as follows:

$$
q=\frac{V_{k}}{R T} p_{k}
$$

The time derivative of the equation (2) can be found:

$$
\frac{d q}{d t}=\frac{V_{k}}{R T} \frac{d p_{k}}{d t}
$$

Because the Saint Venant - Wenzel equations are non-linear, calculation of difference $q_{w}(t)-q_{\mathrm{ps}}(t)$ requires the linearisation. Hence, linear changes in pressure $p_{k}$ and in the mass flow increases were assumed.

Changes in mass flow $\Delta q_{w}(t)$ and $\Delta q_{\mathrm{ps}}(t)$ can be replaced by the exact differentials:

$$
\begin{gathered}
\Delta q_{w}(t)=\left(\frac{\partial \dot{m}_{1}}{\partial A_{w}}\right) \Delta A_{w}+\left(\frac{\partial \dot{m}_{1}}{\partial p_{z}}\right) \Delta p_{z}+\left(\frac{\partial \dot{m}_{1}}{\partial p_{k}}\right) \Delta p_{k} \\
\Delta q_{\mathrm{ps}}(t)=\left(\frac{\partial \dot{m_{1}}}{\partial A_{\mathrm{ps}}}\right) \Delta A_{\mathrm{ps}}+\left(\frac{\partial \dot{m}_{1}}{\partial p_{b}}\right) \Delta p_{b}+\left(\frac{\partial \dot{m}_{1}}{\partial p_{k}}\right) \Delta p_{k}
\end{gathered}
$$

When the equations (3), (4) and (5) are put into the equation (2), after simplification it takes the form:

$T \frac{d \Delta p_{k}}{d t}+\Delta p_{k}=k_{1} \Delta p_{z}+k_{2} \Delta p_{b}+k_{3} \Delta A_{w}+k_{4} \Delta A_{\mathrm{ps}}$

Typically, during the short time of a single measurement, both feeding pressure $p_{z}$ and the outer pressure $p_{b}$ do not change substantially, so they can be assumed constant. Similarly, the cross section of the inlet nozzle $F_{w}$ is constant, so the coefficients $k_{1}, k_{2}, k_{3}$ and $k_{4}$ are equal to zero. As a result, the equation (6) has the form:

$$
T \frac{d \Delta p_{k}}{d t}+\Delta p_{k}=k \Delta F_{\mathrm{ps}}
$$

From the above equation (7), when assuming zero initial conditions, the frequency response (gain) of the air gauges can be written as follows: 


$$
G(s)=\frac{p_{k}(s)}{F_{\mathrm{ps}}(s)}=\frac{k}{T s+1}
$$

The above transfer function describes an air gauge as an inertial first-order dynamical system. It is defined by two parameters: time constant $T$ and multiplication $k$. The time constant $T$ is a characteristic of the air gauge dynamics, while $k$ is the sensitivity or multiplication. The lower value of time constant $T$, the more favourable dynamic properties of the system are.

In the analysed test rig, the amplitude of the backpressure in the air gauge varies according to the equation [18]:

$$
A_{p k}\left(f_{i}\right)=\frac{k}{\sqrt{1+\left(f_{i} T\right)^{2}}}
$$

The equation (9) was applied as a basis for the amplitude-frequency calculations. During the dynamic calibration of the air gauges with various inlet nozzles $d_{w}$, measuring nozzles $d_{p}$, and measuring chamber volumes $V_{k}$, the sine input was generated with a series of frequencies. The registered normalised amplitudes were:

$$
A_{u}\left(f_{i}\right)=\frac{\Delta p_{k_{i}}}{\Delta p_{k(f=0)}}
$$

where:

$\Delta p_{k i}$ - change in back-pressure value corresponding with $i$-th value of applied frequency $f_{i}$;

$\Delta p_{k(f=0)}$ - change in back-pressure value for the static measurement, when $f_{0}=0 \mathrm{~Hz}$.

Figure 3 presents an example of the response of the air gauge derived from the registered points.

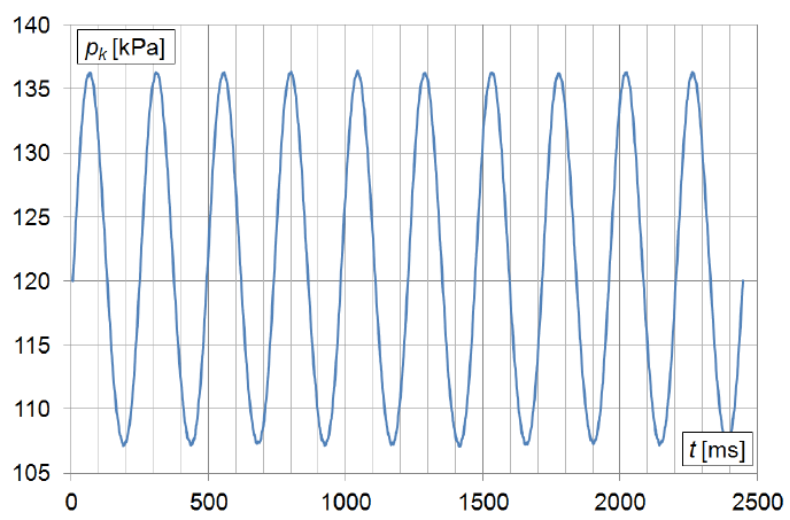

Fig. 3. Example of the air gauge sine input response based on the registered points.

\section{Calculation algorithms}

From the obtained data, the time constants were calculated using the well-known formula [19]:

$$
T=\frac{\sum_{i=1}^{n} \frac{\left(1-A_{u_{i}}^{2}\left(f_{i}\right)\right)}{A_{u_{i}}^{2}\left(f_{i}\right)}}{\sum_{i=1}^{n} f_{i} \frac{\sqrt{1-A_{u_{i}}^{2}\left(f_{i}\right)}}{A_{u_{i}}\left(f_{i}\right)}} .
$$

Then, the frequency $f_{0.95}$ that generated dynamic error $\delta(\omega)=5 \%$ was calculated for each set of the collected data. Perfect reproduction of the input signal is not possible, so some dynamic error is inevitable. It was assumed that the acceptable dynamic error, represented by the amplitude reduction, was $5 \%$.

In order to assess the approximation error, its mean square value $\delta A_{u}$ was calculated from the formula [20]:

$$
\delta A_{u}=\sqrt{\frac{1}{n} \sum_{i=1}^{n}\left(A_{u_{i}}-A_{u t_{i}}\right)^{2}} \cdot 100 \%
$$

where: $i$-th normalised amplitude of the experimental characteristics,

$A_{u i}$ - $i$-th normalised amplitude of the experimental characteristics,

$A_{u t i}$ - $i$-th normalised amplitude of the theoretical characteristics.

The set of registered measuring points after each measurement series underwent the processing as follows [5]:

- smoothening procedure,

- determination of the frequency of the registered sinusoidal signal,

- determination of the amplitudes of the registered signal,

- frequency-amplitude characteristics,

- approximation of the characteristics into the coordinate system,

- time constant calculation.

The smoothening procedure, similarly as in the case of cubic smoothening spline procedure, required the continuity of splined functions $S(x)$ and their derivatives $S^{\prime}(x)$ and $S^{\prime \prime}(x)$. In the formula (13) below, $\lambda$ is a parameter of smoothness, while $\delta_{i}$ values correspond with standard deviations of the measurement error of each obtained point $f_{i}(\mathrm{i}=1,2, \ldots, \mathrm{N})[5]$ :

$$
\sum\left(\frac{f_{i}-S\left(x_{i}\right)}{\delta_{i}}\right)^{2}+\lambda \int_{x 1}^{x N}\left[S^{\prime \prime}(x)\right]^{2} d x=\min
$$

An example of the smoothening of the obtained signal $p_{k}$ is shown in Fig. 4.

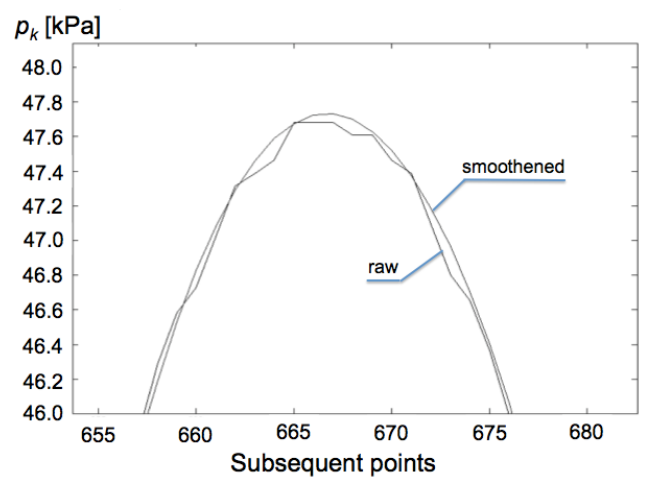

Fig. 4. Example of the smoothening of obtained characteristics.

For each given input frequency $f_{i}$, response signal frequency $f_{p k i}$ and amplitude $A_{p k}\left(f_{i}\right)$ is calculated. The smoothened sinusoid response frequency is determined from the points where it crosses the reference axis. The algorithm calculates the number of measuring points and 
multiplies it by the known sampling frequency $f_{s}$. For each input frequency $f_{i}$, the procedure is repeated.

Subsequently, local maximums and minimums of the registered signal are defined for each frequency. The local extremes are identified using the numerical derivative test. After the extremes are pointed out, their values undergo the averaging procedure and average signal amplitude is calculated. The algorithm is repeated in a loop for each given frequency $f_{i}$ of the sine input signal. The obtained results were compared to the ones obtained from an oscilloscope, which confirmed the correctness of the procedures.

Experimental results of the amplitude-frequency characteristics were compared to the theoretical ones in order to determine the approximation error. The theoretical curve is represented by the following formula:

$$
A(\omega)=\frac{1}{\sqrt{1+T^{2} \omega^{2}}},
$$

Figure 5 demonstrates an example of the experimental and theoretical amplitude-frequency characteristics. The maximal difference between two curves constitutes the approximation error $\delta_{a p p}$, which in the example is approx. $4 \%$. In the series of experiments, the maximal approximation error was $\delta_{a p p \max }=7 \%$, which can be considered a very good result.

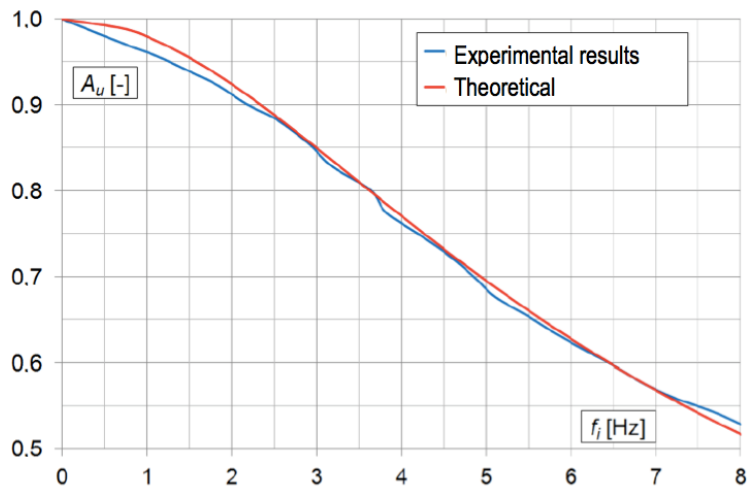

Fig. 5. Example of the amplitude-frequency characteristics obtained from the test rig.

\section{Conclusions}

The described test rig for sine input calibrations of air gauges is fully computerised. The program sets the rotational speed of the eccentric shaft that generates cyclic changes of the measuring slot. The respective back-pressure signal is registered, and the obtained points undergo further processing.

Processing algorithms smoothen and approximate the obtained data so that the final characteristics provide the basis for further calculations. From the amplitudefrequency graph, the time constant can be calculated, as well as the maximal frequency $f_{0.95}$ that generates the dynamic error below $5 \%$.

The back-pressure air gauges with small measuring chambers and piezoresistive pressure transducers perform as the first-order dynamic systems. The algorithms provide results with an approximation error $\delta_{a p p \max }=7 \%$, so the test rig may be successfully used for the dynamic calibration of the air gauges.

\section{References}

1. C.J. Tanner, Institution of Production Engineers Journal, 37, 448 (1958)

2. M.M. Khonsari, E.R. Booser, Applied Tribology (Wiley, Chichester, 2017)

3. A.A. Pleshakov, M.G. Kristal, Meas. Tech. 59, 28 (2016)

4. M. Rucki, B. Barisic, Measurement 43, 83 (2010)

5. J. Jozwik, M. Czwarnowski, Adv. Sci. Technol. Res. J. 9, 89 (2015)

6. J. Jozwik, L. Semotiuk, I. Kuric, Adv. Sci. Technol. Res. J. 9, 96 (2015)

7. H.F. Walker, D.W. Benbow, A.K. Elshennawy, The Certified Quality Technician Handbook (Quality Press, Milwaukee, 2012)

8. Cz. J. Jermak, Acta Mechanica et Automatica 11, 38 (2017)

9. M. Rucki, Cz.J. Jermak, J. Dyn. Sys. Meas. and Cont. 134(1), 011001 (2012)

10. Cz.J. Jermak, Arch. Mech. Technol. Autom. 31(4), 79 (2011)

11. Cz.J. Jermak, R. Majchrowski, Proceedings of the $13^{\text {th }}$ National and $4^{\text {th }}$ International Conference Metrology in Production Engineering, Poznan Zerkow, 2009, p. 201

12. J. Liu, X. Pan, G. Wang, A. Chen, Flow Meas. Inst. 23, 26 (2012)

13. R. Majchrowski, Int. J. Mater. Prod. Technol. (to be published, DOI: 10.1504/IJMPT.2018.10015573)

14. R.S. Figliola, D.E. Beasley, Theory and Design for Mechanical Measurements (Wiley, Clemson, 2011)

15. M. Jakubowicz, Mechanik 11, 1168 (2016)

16. T. Yandayan, M. Burdekin, Intl. J. Machine Tools Manuf. 37(10), 1423 (1997)

17. M. Jakubowicz, M. Rucki, G. Varga, R. Majchrowski, Lecture Notes in Mechanical Engineering, Issue 201519 (2018) Pages 733-742 (5th International Scientific-Technical Conference on Advances in Manufacturing, MANUFACTURING 2017; Poznan; Poland)

18. O.B. Balakšin, Automation of the air gauge dimensional control in mechanical engineering, (Mašinostroenie, Moscow, 1964)

19. R.J. Soboczyński, Investigations on the metrological characteristics of the high-pressure air gauges, (unpublished doctoral thesis, Wrocław, 1977) (in Polish)

20. A. Zięba, Analysis of the measurement data in exact sciences and technics (PWN, Warsaw, 2014) (in Polish 